\title{
Fifty years of preventing and treating childhood behaviour disorders: a systematic review to inform policy and practice
}

\author{
Charlotte Waddell, Christine Schwartz, Caitlyn Andres, Jenny Lou Barican, Donna Yung
}

Faculty of Health Sciences, Children's Health Policy Centre, Simon Fraser University, Vancouver, British Columbia, Canada Correspondence to Dr Charlotte Waddell, Faculty of Health Sciences, Children's Health Policy Centre, Simon Fraser University, VancouverV6B5K3, Canada; charlotte_waddell@sfu.ca

\begin{abstract}
Question Oppositional defiant and conduct disorders (ODD and CD) start early and persist, incurring high individual and collective costs. To inform policy and practice, we therefore asked: What is the best available research evidence on preventing and treating these disorders?

Study selection and analysis We sought randomised controlled trials (RCTs) evaluating interventions addressing the prevention or treatment of behaviour problems in individuals aged 18 years or younger. Our criteria were tailored to identify higher-quality RCTs that were also relevant to policy and practice. We searched the CINAHL, ERIC, MEDLINE, PsycINFO and Web of Science databases, updating our initial searches in May 2017. Thirtyseven RCTs met inclusion criteria_-evaluating 15 prevention programmes, 8 psychosocial treatments and 5 medications. We then conducted narrative synthesis.

Findings For prevention, 3 notable programmes reduced behavioural diagnoses: Classroom-Centered Intervention; Good Behavior Game; and Fast Track. Five other programmes reduced serious behaviour symptoms such as criminal activity. Prevention benefits were long term, up to 35 years. For psychosocial treatment, Incredible Years reduced behavioural diagnoses. Three other interventions reduced criminal activity. Psychosocial treatment benefits lasted from 1 to 8 years. While 4 medications reduced post-test symptoms, all caused important adverse events.

Conclusions Considerable RCT evidence favours prevention.

Clinical implications Effective prevention programmes should therefore be made widely available. Effective psychosocial treatments should also be provided for all children with ODD/CD. But medications should be a last resort given associated adverse events and given only short-term evidence of benefits. Policymakers and practitioners can help children and populations by acting on these findings.
\end{abstract}

\section{BACKGROUND}

Oppositional defiant and conduct disorders (ODD and CD) are important public health and children's mental health problems. They involve severe and persistent patterns of disruptive behaviour that fall well outside expected norms and that interfere with healthy development, with CD being the more serious condition. ${ }^{12}$ These 2 mental disorders are important, in part, because of their high prevalence. Globally, recent estimates suggest rates of $3.6 \%$ for $\mathrm{ODD}$ and $2.1 \%$ for $\mathrm{CD}^{3}$ Disruptive behaviours have also long been a leading reason for referrals to children's mental health services. ${ }^{4}$ As well, because these disorders typically start in childhood then persist into adulthood, they cause inordinate burdens. ${ }^{5}$ The children experience significant social and emotional impairments, with concomitant distress and lost human potential. ${ }^{6}$ Society also incurs substantial healthcare, justice, child welfare and education costs - such that preventing just 1 case of $C D$ at birth may yield lifetime savings of as much as $\$ 3.0-5.1 \mathrm{M}$, based on cost analyses across multiple sectors in the USA (USD, 2017 equivalency). ${ }^{7}$

From a children's rights perspective, prevention should be the highest priority given that behaviour disorders have been causally associated with serious but avoidable adversities such as family socioeconomic disadvantage and child maltreatment. ${ }^{6-10}$ In effect, when avoidable cases are allowed to develop, children are doubly disadvantaged, experiencing both the causes and consequences of behaviour disorders. ${ }^{11}$ Many prevention programmes for ODD/CD have also been shown to be cost-effective. ${ }^{12}$ Yet programmes for the prevention of these disorders - or of their underlying causal conditions such as child maltreatment-have yet to become widespread. $^{13-15}$

Exacerbating the burdens, there are also acute shortages of children's mental health treatment services. Even in high-income countries such as the UK, USA and Canada, as many as $70 \%$ of young people with mental disorders do not receive needed specialised treatment services. ${ }^{16}$ This is despite substantial (and growing) annual health expenditures in these jurisdictions. ${ }^{17-19}$ Globally, the situation is much worse. Many low-income countries have yet to place children's mental health on the public policy agenda, and mental health service shortfalls in these countries reach or exceed $90 \%{ }^{20-22}$ Amid these shortfalls, one approach nevertheless appears to be thriving, at least in high-income countries - the use of pharmacological treatments. For example, psychotropic prescriptions for children have increased approximately twofold in the UK and three-tofourfold in Canada in recent decades, particularly for antipsychotics which are increasingly being used to treat behaviour disorders. ${ }^{23-26}$

To begin to address the shortfalls and imbalances, policymakers need robust research evidence to guide public spending priorities. In parallel, practitioners need robust research evidence to inform the implementation of more effective programmes and services for children.

\section{OBJECTIVE}

To inform policy and practice, we therefore asked: what is the best available research evidence on preventing and treating ODD and CD? To provide comprehensive information, we included the full intervention continuum: prevention programmes, psychosocial treatments and pharmacological treatments. We were aware of only 1 such previous comprehensive review of ODD/CD interventions, Connor et al (2006), ${ }^{27}$ which covered studies published from 1980 to 2005 . We specifically built on this review, covering older studies (published from 1965 onwards) as well as newer studies (published from 2005 through 2017). We also expanded on previous reviews that examined only prevention (eg, Waddell et al 2007), ${ }^{28}$ only treatment (eg, National Collaborating Centre for Mental Health, 2013) ${ }^{29}$ or only specific types of interventions (eg. Woolfenden et al, 2002; Barlow et al, 2011; Sanders et al, 2014; Bakker et al, 2017). ${ }^{30-33}$ To our knowledge, no recent systematic review has covered this comprehensive range of ODD/CD interventions.

\section{STUDY SELECTION AND ANALYSIS}

We first searched the CINAHL, ERIC, MEDLINE and PsycINFO databases using the terms: conduct disorder, oppositional defiant disorder, child behaviour disorder, aggressive behaviour OR juvenile delinquency AND prevention, intervention OR treatment. To identify additional studies, we searched the journal, Evidence-Based Mental Health, and searched the Cochrane and Campbell Collaboration databases, identifying relevant 


\begin{tabular}{|c|c|}
\hline Table 1 & \\
\hline 1 & $\begin{array}{l}\text { Children } \leq 18 \text { years of age were the main focus or were clearly reported } \\
\text { on separately if part of an adult study. }\end{array}$ \\
\hline \multirow[t]{3}{*}{2} & $\begin{array}{l}\text { Interventions addressed childhood behaviour problems, including ODD } \\
\text { and/or CD. }\end{array}$ \\
\hline & $\begin{array}{l}\text { a. For prevention, at enrolment/pretest, }<50 \% \text { had a primary } 0 \mathrm{DD} / \mathrm{CD} \\
\text { diagnosis or had been referred for treatment for behaviour problems or } \\
\text { had been arrested. }\end{array}$ \\
\hline & $\begin{array}{l}\text { b. For treatment, at enrolment/pretest, } \geq 50 \% \text { had a primary } 0 D D / C D \\
\text { diagnosis or had been referred for treatment for behaviour problems or } \\
\text { had been arrested. }\end{array}$ \\
\hline 3 & $\begin{array}{l}\text { Clear descriptions were provided of participant characteristics, study } \\
\text { settings and interventions. }\end{array}$ \\
\hline 4 & $\begin{array}{l}\text { Participants (or clusters) were randomly assigned to intervention and } \\
\text { either control (no-intervention) or comparison (treatment-as-usual) } \\
\text { groups at study outset. }\end{array}$ \\
\hline 5 & $\begin{array}{l}\text { Behaviour indicators included either one diagnostic measure where the } \\
\text { diagnostician was blinded, or two symptom measures evaluated by two } \\
\text { or more informant sources, eg, child, parent or teacher, at least one of } \\
\text { whom was blinded. }\end{array}$ \\
\hline 6 & $\begin{array}{l}\text { Outcome measures pertained to } \mathrm{ODD} / \mathrm{CD} \text {, eg, scales had established } \\
\text { reliability and validity or } \geq 50 \% \text { of items addressed } \mathrm{ODD} / \mathrm{CD} \text { symptoms. }\end{array}$ \\
\hline 7 & $\begin{array}{l}\text { Maximum attrition was } 20 \% \text { at post-test (medication studies) or at } \\
\text { follow-up (prevention or psychosocial treatment studies) or authors used } \\
\text { intention-to-treat analyses. }\end{array}$ \\
\hline 8 & $\begin{array}{l}\text { For prevention and psychosocial treatment studies, postintervention } \\
\text { follow-up was } 3 \text { months or more. }\end{array}$ \\
\hline 9 & $\begin{array}{l}\text { For medication studies, double-blinding and placebo controls were used, } \\
\text { and side effects were comprehensively assessed. }\end{array}$ \\
\hline 10 & $\begin{array}{l}\text { Statistical significance (using } \mathrm{p}<0.05 \text { ) was reported for relevant } \\
\text { outcome measures at post-test (medication studies) or at follow-up } \\
\text { (prevention and psychosocial treatment studies). }\end{array}$ \\
\hline 11 & $\begin{array}{l}\text { Interventions were evaluated in high-income countries (by World Bank } \\
\text { standards). }\end{array}$ \\
\hline 12 & $\begin{array}{l}\text { Studies were not limited to specialised populations, such as children } \\
\text { residing in abused women's shelters, to retain broad applicability to } \\
\text { children at risk of or who have ODD/CD. }\end{array}$ \\
\hline
\end{tabular}

*For inclusion, all criteria had to be met.

$\mathrm{CD}$, conduct disorder; $\mathrm{ODD}$, oppositional defiant disorder.

systematic reviews that we then hand-searched. We applied limiters, seeking only randomised controlled trials (RCTs) evaluating interventions addressing the prevention or treatment of behaviour problems in individuals aged 18 years or younger. We limited our searches to English-language articles due to most research being published in this language and due to translation capacity not being available within the team. We also chose to seek articles published over the past 50 years (January 1965 through May 2017, which was the time of the last search update) to ensure comprehensiveness while also ensuring complete coverage of important databases such as MEDLINE and ERIC, which began in 1966. Following these searches, after screening the titles, 2 authors independently assessed all relevant abstracts to identify eligible RCTs. Subsequently, 2 authors independently assessed all retrieved articles, identifying RCTs that met all the inclusion criteria. We then identified supplemental publications on these RCTs by searching the Web of Science database using intervention names, author names and article titles. This added search yielded new follow-up data from the previously identified RCTs as well as new RCTs that were assessed using the procedures noted above. Table 1 shows the inclusion criteria. Figure 1 shows the search process.

We took several steps to maximise quality and minimise risk of bias. To ensure high-quality evaluations, only RCTs were included. Our inclusion criteria also specified additional quality indicators, namely blinding of at least 1 informant source for psychosocial studies and

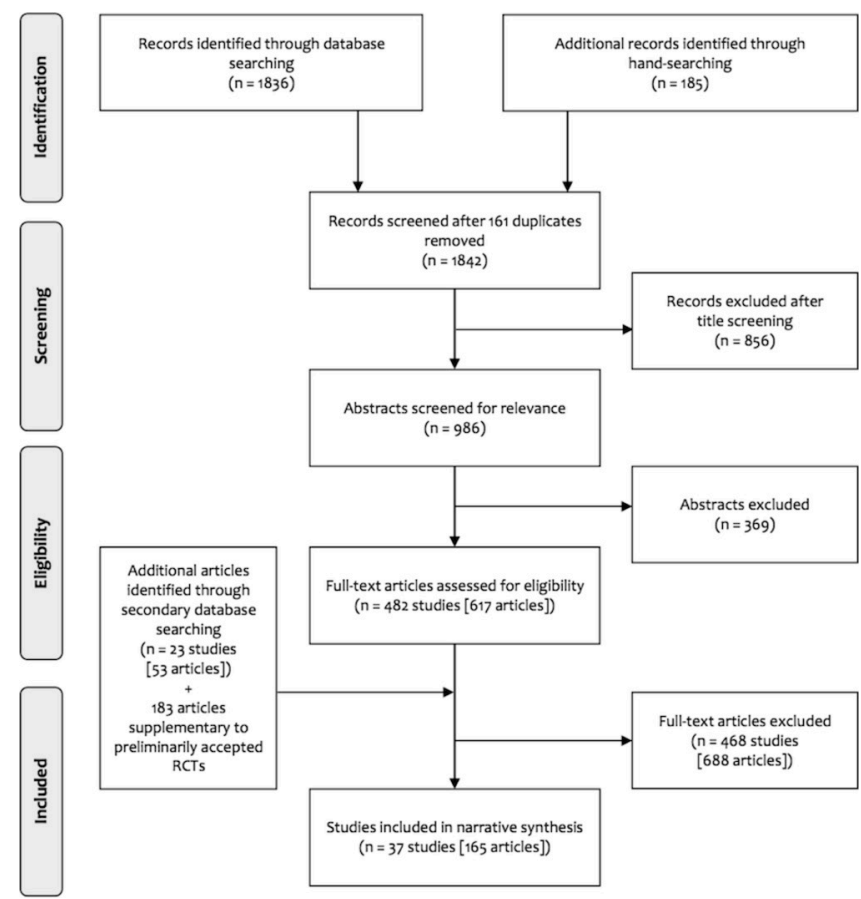

Figure 1 Search process adapted from CONSORT flow diagram. ${ }^{47}$

double-blinding for medication studies. To minimise risk of bias, 2 or more authors independently verified search results, screened abstracts and assessed retrieved articles. At each step, disagreements were resolved by consensus. Data extraction, verification, analysis and interpretations were also performed using consensus. To maximise policy and practice applicability, we focused on studies conducted in high-income countries, given that low-income countries have yet to be able to mobilise children's mental health services on any large scale. ${ }^{20-22}$ This approach yielded 37 RCTs (described in 165 articles) that met inclusion criteria - reporting on 15 prevention programmes, 8 psychosocial treatments and 5 pharmacological treatments. We then assessed the risk of bias for each of these 37 RCTs using the Cochrane risk-of-bias tool. ${ }^{34}$

Due to heterogeneity in both participants and interventions in the included studies, a meta-analysis was not conducted. Rather, we structured a narrative synthesis according to intervention types and outcomes. Interventions were first categorised as prevention programmes, psychosocial treatments or pharmacological treatments. Behaviour findings pertaining to ODD/CD were then extracted, including long-term outcomes for related adult conditions such as antisocial personality disorder (ASPD). Diagnostic findings were extracted for all follow-up periods. Symptom findings were extracted for the longest available follow-up period or for 2 follow-up periods when needed to meet the criterion of reporting on 2 or more behaviour symptoms (including 1 that was blinded). (This latter step allowed us to include a broader range of interventions of relevance to policy and practice, while still ensuring rigour.)

Next, we evaluated the overall quality of the findings. We deemed interventions 'notable' if RCTs showed significant reductions in rates of childhood ODD/CD and/or adult ASPD diagnoses, given that diagnostic outcomes are a particularly robust indicator of effectiveness. We also noted effect sizes, where available, for both diagnostic and behaviour symptom outcomes. Meanwhile, we deemed adverse events associated with medications to be 'important' if they included any of the following symptoms: neurological (dizziness, nausea, vomiting, dystonia, drooling, tremor, headache or diplopia); gastrointestinal (abdominal pain); cardiovascular (tachycardia); endocrine (increased prolactin levels); genitourinary (excessive thirst or excessive urination) and psychiatric (sedation, fatigue, irritability, restlessness or anxiety). This review was registered 
with PROSPERO (registration number CRD42016052643; see www.crd. york.ac.uk/PROSPERO/).

\section{FINDINGS \\ Prevention programmes}

Sixteen RCTs met inclusion criteria, evaluating 15 different prevention

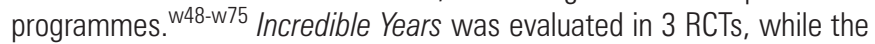
Positive Parenting Program (Triple P) was evaluated in 2 RCTs. As well, 2 RCTs evaluated 2 different programmes: Classroom-Centered Intervention and Family School Partnership, each assessed independently; and Triple $P$ and Promoting Alternative Thinking Strategies (PATHS), each assessed both independently and in combination. Most programmes included parent training. Many also focused on developing children's social skills and/or their academic skills. All programmes were delivered in community settings such as homes, preschools and schools. Three interventions were delivered universally, while 12 were delivered to children at risk or their parents. Intervention duration ranged widely_from 1 month to 10 years.

Thirteen of 15 prevention programmes succeeded in significantly reducing diagnoses or symptoms or both. Three programmes were notable for significantly reducing childhood CD or adult ASPD diagnoses: Good Behavior Game (ASPD diagnoses); ${ }^{\text {w8 }}$ Classroom-Centered Intervention (which included Good Behavior Game; $\mathrm{CD}$ diagnoses, $\mathrm{OR}=0.42$ ); w54 and Fast Track (ASPD diagnoses, $\mathrm{OR}=0.60$ ). ${ }^{\text {w63 }}$

As well, 5 programmes significantly reduced particularly serious behaviour symptoms, namely engagement in criminal activities, arrests or days incarcerated. These programmes included: Coping Power (criminal activities, Cohen's $d[d]=0.27$ ): ${ }^{\text {w57 }}$ Fast Track (convictions/diversions for violent and substance-related crimes); w63 Nurse-Family Partnership (arrests, convictions and probation violations and adjudications as a being in need of supervision): w70 Parent Management Training-Oregon (arrests, $d=0.28$ and younger age at first arrest); $; 71$ and Perry Preschool (arrests, $\mathrm{OR}=0.54$ and months sentenced to prison, $\mathrm{OR}=0.48)$. ${ }^{\mathrm{w} 72}$

Six additional programmes significantly reduced at least 1 behaviour symptom. These included: Chicago Parent Program (behaviour problems): w55 Family Check-Up (behaviour problems); w60 Family School Partnership (behaviour problems, $d=0.29$ and school suspensions,

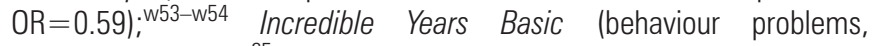
$d=0.63$ and 0.89$)^{\text {w65 }}$ and coupled with Incredible Years Enhanced (aggression):"w67 Research-Based Developmentally Informed (REDI) program (behaviour problems, $d=0.25$ ); ${ }^{\text {w74 }}$ and Triple $P$ / (behaviour problems) ${ }^{\mathrm{w} 49}$ and // (behaviour problems and aggression, $d=0.11$ and 0.15 , respectively). ${ }^{\text {w51 }}$ Only 2 interventions failed to show any behaviour benefits at relevant follow-up: Montreal Prevention Program ${ }^{\text {w68-w69 }}$ and PATHS. ${ }^{\text {w51 }}$ Table 2 summarises the 15 programmes and their evaluations.

Reported effect sizes varied, as noted above. For reductions in diagnoses, these ranged from $\mathrm{OR}=0.42$ (for Classroom-Centered Intervention) to $\mathrm{OR}=0.60$ (for Fast Track). For reductions in criminal behaviours, these ranged from $d=0.27$ (for Coping Power) to $\mathrm{OR}=0.48$ (for Perry Preschool). Effect sizes for reductions in general behaviour symptoms, meanwhile, ranged from $d=0.11$ (for Triple $P$ II) to $d=0.89$ (for Incredible Years Basic). These numbers suggest modest-to-moderately robust effects overall. Adverse events were not reported for any of the included prevention programmes.

\section{Psychosocial treatments}

Thirteen RCTs met inclusion criteria, evaluating 8 different psychosocial treatments. ${ }^{\text {w76-w93 }}$ All included parents in at least 1 version of the intervention. Five also intervened directly with children, eg, providing cognitive-behavioural therapy or social skills training. Some also provided family therapy. Treatments were delivered in clinics as well as homes, schools and other community settings. One intervention involved placing youth in specialised foster homes. Interventions were relatively shortranging from 2 weeks to 7 months.

Five of 8 psychosocial treatments significantly reduced diagnoses or symptoms or both. One treatment-Incredible Years - was notable for significantly reducing ODD diagnoses. Incredible Years Basic reduced ODD diagnoses at 7.8 year (final) follow-up $(\mathrm{OR}=0.20)$. ${ }^{\text {w77 }}$ Incredible Years Basic Plus Literacy also reduced ODD diagnoses at 4-month follow-up $(\mathrm{OR}=0.30)$; ${ }^{\mathrm{w} 79}$ however, by 5.8-year (final) follow-up, this outcome was no longer statistically significant. ${ }^{\text {w77 }}$ Three programmes stood out for significantly reducing particularly serious behaviour symptoms, namely engagement in criminal activities or days incarcerated. These treatments included: Brief Intervention-Youth Only (arrests):"w76 Multidimensional Treatment Foster Care / (violent offences) ${ }^{\text {w80 }}$ and // (days incarcerated): ${ }^{\text {w82 }}$ and Multisystemic Therapy // (criminal activities, $\mathrm{OR}=0.41$ ) w84 $^{2}$ and $I V$ (property offences, $d=0.37$ ). ${ }^{\text {w87 }}$ One other programme, ParentChild Interaction Therapy, reduced 1 (non-criminal) symptom (behaviour problems, $d=0.61-0.64)$. ${ }^{\text {} 90}$ The remaining 3 programmes-Parent Management Training-Oregon, Protocol for Onsite Nurse-Administered Intervention and Strongest Families — failed to produce positive behaviour outcomes at relevant follow-up. ${ }^{\text {w91-w93 }}$ Table 3 summarises the 8 psychosocial treatments and their evaluations.

Reported effect sizes varied, as noted above. For reductions in diagnoses, effect size was $\mathrm{OR}=0.20$ (for Incredible Years Basic). For reductions in criminal behaviour, effect sizes ranged from $d=0.37$ (for Multisystemic Therapy IV) to $\mathrm{OR}=0.41$ (for Multisystemic Therapy II). The one programme that reduced general behaviour symptoms had effect sizes of $d=0.61-0.64$ (for Parent-Child Interaction Therapy). These numbers suggest modest-to-moderately robust effects overall. Adverse events were not reported for any of the included psychosocial treatments.

\section{Pharmacological treatments}

Eight RCTs met inclusion criteria, evaluating 5 different medications: 3 antipsychotics (haloperidol, quetiapine and risperidone), 1 antiepileptic (carbamazepine) and 1 mood stabiliser (lithium).. ${ }^{\text {w9-w101 }}$ RCTs were conducted in inpatient and outpatient settings. All RCTs assessed outcomes at post-test but not beyond, and assessed symptoms but not diagnoses.

All pharmacological treatments succeeded in reducing child behaviour symptoms with the exception of carbamazepine. Two RCTs also provided data on effect sizes, showing large benefits. Specifically, lithium significantly reduced the odds of children experiencing behaviour symptoms $(\mathrm{OR}=9.3)^{\mathrm{w} 97}$ while quetiapine significantly reduced symptom severity $(d=1.6){ }^{\text {w98 }}$ However, adverse events were reported for most children across all the RCTs - 100\% of children in some cases. As well, important adverse events were noted for at least $50 \%$ of intervention children in at least 1 RCT for all medications. These problems included: dizziness (carbamazepine); ${ }^{\text {w94 }}$ sedation and dystonia (haloperidol); ${ }^{25}$ nausea, vomiting, excessive thirst and excessive urination (lithium); ${ }^{\text {w97 }}$ irritability, restlessness, agitation, anxiety and sedation (quetiapine); ${ }^{9} 98$ and fatigue and sedation(risperidone). ${ }^{\text {w9 }}$ w101 Table 4 summarises the 5 pharmacological treatments and their evaluations.

\section{Risk of bias in included studies}

Applying the Cochrane risk-of-bias assessment tool, ${ }^{34}$ we identified the following indicators of risk for our included RCTs. For prevention studies, selection bias was unclear for most; performance bias was high for all; detection bias was low for most; and attrition and reporting biases were low for all. For psychosocial treatment studies, selection bias was unclear for most; performance bias was high for all; detection bias was high for most and attrition and reporting biases were low for all. For medication studies, meanwhile, selection bias was unclear for most, while performance, detection, attrition and reporting biases were low for all. The overall risk-of-bias profile was therefore more favourable for medication 
Table 2 Prevention programme descriptions and evaluation findings

\begin{tabular}{|c|c|c|c|c|c|c|}
\hline Programme & $\begin{array}{l}\text { Sample size } \\
\text { (country) }\end{array}$ & $\begin{array}{l}\text { Ages/Grades at } \\
\text { programme start } \\
\text { (risk factors) }\end{array}$ & $\begin{array}{l}\text { Programme } \\
\text { elements } \\
\text { (settings) }\end{array}$ & $\begin{array}{l}\text { Programme } \\
\text { duration }\end{array}$ & $\begin{array}{l}\text { Follow-up } \\
\text { after post-test }\end{array}$ & $\begin{array}{l}\text { Child } \\
\text { behaviour outcomes* }\end{array}$ \\
\hline \multicolumn{7}{|l|}{ Universal programmes } \\
\hline Good Behavior Game ${ }^{\mathrm{w} 48}$ & 922 (USA) & $\begin{array}{l}\text { Grade } 1 \\
\text { (not applicable) }\end{array}$ & $\begin{array}{l}\text { Child BT } \\
\text { (school) }\end{array}$ & 2 school years & 14 years & $\downarrow$ ASPD diagnoses \\
\hline Triple $P / /^{\text {w49-w50 }}$ & $\begin{array}{l}280 \\
\text { (Germany) }\end{array}$ & $\begin{array}{l}3-6 \text { years } \\
\text { (not applicable) }\end{array}$ & $\begin{array}{l}\text { Parent training } \\
\text { (community) }\end{array}$ & 1 month & $\begin{array}{l}3.9 \text { years } \\
11 \text { months }\end{array}$ & $\begin{array}{l}\downarrow 2 \text { of } 8 \text { symptoms } \\
\text { - } 1 \text { of } 1 \text { symptom }\end{array}$ \\
\hline Triple P II alone & $\begin{array}{l}1675 \\
\text { (Switzerland) }\end{array}$ & $\begin{array}{l}\text { Grade } 1 \text {-Triple P II } \\
\text { (not applicable) }\end{array}$ & $\begin{array}{l}\text { Parent training } \\
\text { (community) }\end{array}$ & 1 month & 8 years & $\downarrow 2$ of 7 symptoms $\dagger$ \\
\hline PATHS alone & & $\begin{array}{l}\text { Grade 2-PATHS } \\
\text { (not applicable) }\end{array}$ & $\begin{array}{l}\text { Child SST } \\
\text { (school) }\end{array}$ & 1 school year & 7 years & - 7 of 7 symptoms $\dagger$ \\
\hline Triple P II+PATHS ${ }^{\mathrm{w} 51-\mathrm{w} 52}$ & & As above & As above & As above & 7 years & - 7 of 7 symptoms $\dagger$ \\
\hline \multicolumn{7}{|l|}{ Targeted programmes } \\
\hline \multirow{2}{*}{$\begin{array}{l}\text { Classroom-Centered } \\
\text { Intervention } \ddagger\end{array}$} & \multirow[t]{4}{*}{653 (USA) } & \multirow{4}{*}{$\begin{array}{l}\text { Grade } 1 \\
\text { (low income) }\end{array}$} & \multirow{2}{*}{$\begin{array}{l}\text { Child enriched curriculum, child SST + child BT } \\
\text { (school) }\end{array}$} & \multirow[t]{4}{*}{1 school year } & $5-11$ years§ & $\downarrow 1$ of 1 symptom \\
\hline & & & & & 5 years & $\begin{array}{l}\downarrow \text { CD diagnoses } \\
\downarrow 2 \text { of } 2 \text { symptoms }\end{array}$ \\
\hline \multirow{2}{*}{$\begin{array}{l}\text { Family School } \\
\text { Partnership }{ }^{\text {w53-w54 }}\end{array}$} & & & \multirow[t]{2}{*}{ Parent training + parent-school collaboration (school) } & & $5-11$ years§ & $\downarrow 1$ of 1 symptom \\
\hline & & & & & 5 years & $\begin{array}{l}- \text { CD diagnoses } \\
\downarrow 2 \text { of } 2 \text { symptoms }\end{array}$ \\
\hline $\begin{array}{l}\text { Chicago Parent } \\
\text { Program }^{\text {w55 }}\end{array}$ & 504 (USA) & $\begin{array}{l}2-4 \text { years } \\
\text { (low income) }\end{array}$ & $\begin{array}{l}\text { Parent training } \\
\text { (community) }\end{array}$ & 2.8 months & 1 year & $\downarrow 2$ of 4 symptoms \\
\hline \multirow[t]{2}{*}{ Coping Power ${ }^{\mathrm{w} 56-\mathrm{w} 58 \pi}$} & \multirow[t]{2}{*}{245 (USA) } & \multirow{2}{*}{$\begin{array}{l}\text { Grade } 5 \\
\text { (behaviour problems) }\end{array}$} & \multirow{2}{*}{$\begin{array}{l}\text { Parent training + child CBT } \\
\text { (school) }\end{array}$} & \multirow[t]{2}{*}{2 school years } & 3.5 years & $\downarrow 1$ of 1 symptom \\
\hline & & & & & 1 year & $\downarrow 1$ of 1 symptom** \\
\hline \multirow[t]{2}{*}{ Family Check-Up w59-w62 } & \multirow[t]{2}{*}{731 (USA) } & \multirow{2}{*}{$\begin{array}{l}2 \text { years (behaviour problems, } \\
\text { maternal challenges +/or low SES) }\end{array}$} & \multirow{2}{*}{$\begin{array}{l}\text { Parent training } \\
\text { (home) }\end{array}$} & \multirow[t]{2}{*}{4.3 years } & 4.2 years & - 1 of 1 symptom \\
\hline & & & & & 2.2 years & $\downarrow 1$ of 1 symptom \\
\hline \multirow[t]{2}{*}{ Fast Track ${ }^{\mathrm{w} 63-\mathrm{w} 64} \mathrm{\dagger} \dagger$} & \multirow[t]{2}{*}{891 (USA) } & \multirow[t]{2}{*}{$\begin{array}{l}\text { Grade } 1 \\
\text { (behaviour problems) }\end{array}$} & \multirow[t]{2}{*}{$\begin{array}{l}\text { Parent training, child SST+child tutoring } \\
\text { (school, community, home) }\end{array}$} & \multirow[t]{2}{*}{10 school years } & 8 years & $\begin{array}{l}\downarrow \text { ASPD diagnoses } \\
\downarrow 2 \text { of } 5 \text { symptoms** }\end{array}$ \\
\hline & & & & & 2 years & • ODD/CD diagnoses \\
\hline Incredible Years Basic ${ }^{\mathrm{w} 65}$ & 153 (UK) & $\begin{array}{l}\text { 3-4 years } \\
\text { (behaviour problems) }\end{array}$ & $\begin{array}{l}\text { Parent training } \\
\text { (community) }\end{array}$ & 2.8 months & 3.2 months & $\downarrow 2$ of 4 symptoms \\
\hline $\begin{array}{l}\text { Incredible Years } \\
\text { Basic + Literacy }\end{array}$ & 174 (UK) & $\begin{array}{l}5-6 \text { years } \\
\text { (low income) }\end{array}$ & $\begin{array}{l}\text { Parent training + child literacy training } \\
\text { (community) }\end{array}$ & 4.1 months & 7.9 months & - 4 of 4 symptoms \\
\hline $\begin{array}{l}\text { Incredible } \\
\text { Years Enhanced }{ }^{\mathrm{w67}}\end{array}$ & 92 (USA) & $\begin{array}{l}2-5 \text { years } \\
\text { (sibling in justice system) }\end{array}$ & $\begin{array}{l}\text { Parent training } \\
\text { (community, home) }\end{array}$ & 9-11 months & 8 months & $\downarrow 1$ of 2 symptoms \\
\hline \multirow{2}{*}{$\begin{array}{l}\text { Montreal Prevention } \\
\text { Program }^{\mathrm{w} 68} \text { w69 }\end{array}$} & \multirow[t]{2}{*}{250 (Canada) } & Grade 2 & Child SST, child BT, parent training + teacher training & 2 school years & 19 years & - 1 of 1 symptom \\
\hline & & & (school, home) & & 15 years & - 1 of 1 symptom \\
\hline Nurse-Family Partnership w70 & 400 (USA) & $\begin{array}{l}\text { Prenatal } \\
\text { (low income, first-time mothers) }\end{array}$ & $\begin{array}{l}\text { Parent training } \\
\text { (home) }\end{array}$ & $\begin{array}{l}26 \text { months } \\
\text { (average) }\end{array}$ & 13 years & $\downarrow 3$ of 14 symptoms $* *$ \\
\hline $\begin{array}{l}\text { Parent-Management } \\
\text { Training-Oregon }{ }^{\text {w71 }}\end{array}$ & 238 (USA) & $\begin{array}{l}6-10 \text { years } \\
\text { (boys living with recently separated } \\
\text { mothers) }\end{array}$ & $\begin{array}{l}\text { Parent training } \\
\text { (community) }\end{array}$ & 3.2 months & 8.7 years & $\downarrow 3$ of 3 symptoms** \\
\hline Perry Preschoo/w72 & 128 (USA) & $\begin{array}{l}\text { 3-4 years } \\
\text { (low income+low } 10 \text { ) }\end{array}$ & $\begin{array}{l}\text { Child enriched curriculum + parent-school collaboration } \\
\text { (community, home) }\end{array}$ & 16 months & 35 years & $\downarrow 2$ of 9 symptoms $* *$ \\
\hline$R E D I^{w 73-w 75} \dagger \dagger$ & 356 (USA) & 4 years & Child SST, child literacy training + teacher & 4 months & 4 years & - 1 of 1 symptom \\
\hline & & (low income) & training (communit & & 1 year & $\downarrow 2$ of 2 symptoms \\
\hline
\end{tabular}

$\downarrow$ denotes statistically significant reductions in diagnoses/symptoms; $\bullet$ denotes no significant differences between intervention and control groups.

* Diagnostic findings extracted for all follow-up periods and symptom findings extracted for either longest follow-up period or for two follow-up periods when needed to meet criterion of reporting on 2 or more behaviour symptoms (including one that was blinded).

tOutcome measure completed by teachers during earlier follow-up was blinded so assumed later blinding as well.

$\ddagger$ Good Behavior Game included in Classroom-Centered Intervention.

$\S$ Analysis included follow-up ranging from 5 to 11 years.

TOf 245 children, 123 also received a universal intervention (Coping with Middle School Transitions); outcomes only reported for Coping Power (targeted) because it alone met criteria.

**Reductions included particularly serious symptoms such as criminal activities, arrests, days incarcerated.

†TPATHS included in both Fast Track and REDI.

ASPD, antisocial personality disorder; BT, behaviour training; CBT, cognitive-behavioural training; CD, conduct disorder; ODD, oppositional defiant disorder; PATHS, Promoting Alternative Thinking Strategies; REDI, ResearchBased Developmentally Informed; SES, socioeconomic status; SST, social skills training.

compared with psychosocial studies. Nevertheless, even though many outcome measures for the prevention and psychosocial treatment RCTs were not blinded, a further audit found that more of the blinded outcomes were statistically significant than the non-blinded $142.9 \%$ vs $31.5 \%$, respectively), suggesting that the lack of blinding did not create a bias favouring these types of interventions. Please see the online supplementary appendix for risk-of-bias assessments for individual RCTs.

\section{CONCLUSION AND CLINICAL IMPLICATIONS}

Childhood ODD and CD incur high individual and collective costs, in part because effective prevention and psychosocial treatment interventions are not made widely available. Conducted to encourage improvements in policy and practice, this systematic review identified 37 RCTs evaluating 15 prevention programmes, 8 psychosocial treatments and 5 pharmacological treatments. For prevention, 3 programmes reduced behavioural diagnoses (Classroom-Centered Intervention; Good Behavior Game and Fast Track) while 5 programmes reduced serious behaviour symptoms such as criminal activity (Coping Power; Fast Track; Nurse-Family Partnership, Parent Management Training-Oregon and Perry Preschool). Prevention benefits were long term, up to 35 years. For psychosocial treatment, 1 intervention reduced behavioural diagnoses (Incredible Years) while 3 interventions reduced criminal activity (Brief Intervention; 
Table 3 Psychosocial treatment descriptions and evaluation findings

\begin{tabular}{|c|c|c|c|c|c|c|}
\hline Programme & $\begin{array}{l}\text { Sample size } \\
\text { (country) }\end{array}$ & $\begin{array}{l}\text { Ages at } \\
\text { programme } \\
\text { start }\end{array}$ & $\begin{array}{l}\text { Programme } \\
\text { elements } \\
\text { (settings) }\end{array}$ & $\begin{array}{l}\text { Treatment } \\
\text { duration }\end{array}$ & $\begin{array}{l}\text { Follow-up after } \\
\text { post-test }\end{array}$ & $\begin{array}{l}\text { Child behaviour } \\
\text { outcomes* }\end{array}$ \\
\hline \multirow[t]{2}{*}{ Brief Intervention - Youth only } & \multirow[t]{4}{*}{300 (USA) } & \multirow[t]{4}{*}{$11-17$ years } & \multirow{2}{*}{$\begin{array}{l}\text { Child CBT } \\
\text { (home) }\end{array}$} & \multirow[t]{2}{*}{2 weeks } & 2 years & $\downarrow 1$ of 1 symptomt \\
\hline & & & & & 1.5 years & - 2 of 2 symptoms \\
\hline \multirow{2}{*}{$\begin{array}{l}\text { Brief Intervention- } \\
\text { Youth }+ \text { Parent }\end{array}$} & & & \multirow{2}{*}{$\begin{array}{l}\text { Child CBT + Parent training } \\
\text { (home) }\end{array}$} & \multirow[t]{2}{*}{3 weeks } & 2 years & - 1 of 1 symptom \\
\hline & & & & & 1.5 years & - 2 of 2 symptoms \\
\hline Incredible Years Basic ${ }^{\text {w77-w78 }}$ & 120 (UK) & $3-7$ years & $\begin{array}{l}\text { Parent training } \\
\text { (clinic) }\end{array}$ & 3-3.7 months & 7.8 years (average) & $\begin{array}{l}\downarrow \text { ODD diagnoses } \\
\downarrow 1 \text { of } 3 \text { symptoms }\end{array}$ \\
\hline \multirow[t]{2}{*}{$\begin{array}{l}\text { Incredible Years } \\
\text { Basic + Literacy }\end{array}$} & \multirow[t]{2}{*}{112 (UK) } & \multirow[t]{2}{*}{$4-6$ years } & \multirow[t]{2}{*}{$\begin{array}{l}\text { Parent training + child literacy training } \\
\text { (community, home) }\end{array}$} & \multirow[t]{2}{*}{6.4 months } & $\begin{array}{l}5.8 \text { years } \\
\text { (average) }\end{array}$ & $\begin{array}{l}\text { - ODD diagnoses } \\
\text { - } 3 \text { of } 3 \text { symptoms }\end{array}$ \\
\hline & & & & & 4 months & $\downarrow$ ODD diagnoses \\
\hline $\begin{array}{l}\text { Multidimensional Treatment } \\
\text { Foster Care } I^{\mathrm{w} 80-\mathrm{w} 81} \ddagger\end{array}$ & 79 (USA) & $\begin{array}{l}\text { 12-17 years } \\
\text { (boys only) }\end{array}$ & $\begin{array}{l}\text { Parent training, child SST, family therapy } \\
\text { (home, community) }\end{array}$ & 6.8 months (average) & $\begin{array}{l}1.4 \text { years } \\
\text { (average) }\end{array}$ & $\downarrow 2$ of 2 symptoms $t$ \\
\hline $\begin{array}{l}\text { Multidimensional Treatment } \\
\text { Foster Care } / /^{\mathrm{w} 82} \ddagger\end{array}$ & 81 (USA) & $\begin{array}{l}13-17 \text { years } \\
\text { (girls only) }\end{array}$ & $\begin{array}{l}\text { Parent training, child SST, family therapy } \\
\text { (home, community) }\end{array}$ & 5.7 months (average) & $\begin{array}{l}1.4 \text { years } \\
\text { (average) }\end{array}$ & $\downarrow 1$ of 3 symptomst \\
\hline Multisystemic Therapy I ${ }^{\mathrm{w} 83} \ddagger, \S$ & 118 (USA) & $12-17$ years & $\begin{array}{l}\text { Parent training, child CBT, family therapy } \\
\text { (home, community) }\end{array}$ & 4.3 months (average) & 6 months & - 2 of 2 symptoms \\
\hline Multisystemic Therapy II ${ }^{\mathrm{w} 84} \ddagger$ & 131 (USA) & $11-17$ years & $\begin{array}{l}\text { Parent training, child CBT, family therapy } \\
\text { (home, community) }\end{array}$ & $\begin{array}{l}7 \text { months } \\
\text { (average) }\end{array}$ & $\begin{array}{l}1.4 \text { years } \\
\text { (average) }\end{array}$ & $\downarrow 1$ of 2 symptoms $t$ \\
\hline \multirow{2}{*}{$\begin{array}{l}\text { Multisystemic Therapy } \\
\text { III }{ }^{\text {w85-w86 }}\end{array}$} & \multirow[t]{2}{*}{164 (USA) } & \multirow[t]{2}{*}{$11-18$ years } & \multirow{2}{*}{$\begin{array}{l}\text { Parent training, child CBT, family therapy } \\
\text { (home, community + school) }\end{array}$} & \multirow{2}{*}{$\begin{array}{l}5.2 \text { months } \\
\text { (average) }\end{array}$} & 2 years & - 2 of 2 symptoms \\
\hline & & & & & 1 year & $\downarrow 2$ of 4 symptoms \\
\hline \multirow[t]{2}{*}{$\begin{array}{l}\text { Multisystemic Therapy } \\
\text { IN87-w89 }\end{array}$} & \multirow[t]{2}{*}{$\begin{array}{l}256 \\
\text { (Netherlands) }\end{array}$} & \multirow[t]{2}{*}{$12-18$ years } & \multirow[t]{2}{*}{$\begin{array}{l}\text { Parent training, child CBT, family therapy } \\
\text { (home, community) }\end{array}$} & \multirow[t]{2}{*}{5.7 months (average) } & $\begin{array}{l}2.8 \text { years } \\
\text { (average) }\end{array}$ & - 1 of 1 symptom \\
\hline & & & & & $\begin{array}{l}7.2 \text { months } \\
\text { (average) }\end{array}$ & $\downarrow 5$ of 6 symptoms $\dagger$ \\
\hline $\begin{array}{l}\text { Parent-Child Interaction } \\
\text { Therapy }^{\text {w90 }}\end{array}$ & 81 (Norway) & $2-7$ years & $\begin{array}{l}\text { Parent training } \\
\text { (clinic) }\end{array}$ & $\begin{array}{l}4.9 \text { months } \\
\text { (average) }\end{array}$ & $\begin{array}{l}1.1 \text { years } \\
\text { (average) }\end{array}$ & $\downarrow 2$ of 5 symptoms \\
\hline $\begin{array}{l}\text { Parent Management Training- } \\
\text { Oregon w91 }\end{array}$ & 112 (Norway) & $4-12$ years & $\begin{array}{l}\text { Parent training } \\
\text { (community) }\end{array}$ & 6.1 months (average) & $\begin{array}{l}1.1 \text { years } \\
\text { (average) }\end{array}$ & - 7 of 7 symptoms \\
\hline $\begin{array}{l}\text { Protocol for On-Site Nurse- } \\
\text { Administered Intervention }{ }^{\text {w92 }}\end{array}$ & 163 (USA) & $6-11$ years & $\begin{array}{l}\text { Parent training, child CBT, family therapy } \\
\text { (clinic) }\end{array}$ & 4 months (average) & 1 year & $\begin{array}{l}-0 D D / C D \text { diagnoses } \\
\text { - } 1 \text { of } 1 \text { symptom }\end{array}$ \\
\hline Strongest Families ${ }^{\mathrm{w} 93} \boldsymbol{q}$ & 80 (Canada) & $3-7$ years & $\begin{array}{l}\text { Parent training } \\
\text { (home) }\end{array}$ & 3.2 months & 5.2 months & - ODD diagnoses \\
\hline
\end{tabular}

$\downarrow$ denotes statistically significant reductions in diagnoses/symptoms; $\bullet$ denotes no significant differences between intervention and control groups.

*Diagnostic findings extracted for all follow-up periods and symptom findings extracted for either longest follow-up period or for 2 follow-up periods when needed to meet criterion of reporting on two or more behaviour symptoms (including one that was blinded).

tReductions included particularly serious behaviour symptoms, including criminal activities, arrests and days incarcerated.

$\ddagger$ All youth had prior justice system involvement.

$\S$ All youth also met diagnostic criteria for a substance use disorder.

IIntervention predominately self-delivered using handbooks and videos supplemented with weekly telephone coaching.

$\mathrm{CD}$, conduct disorder; CBT, cognitive-behavioural training; ODD, oppositional defiant disorder; SST, social skills training.

Multidimensional Treatment Foster Care and Multisystemic Therapy). Psychosocial treatment benefits lasted from 1 to 8 years. Meanwhile, 4 medications, mainly antipsychotics, reduced post-test symptoms, yet all caused important adverse events.

We believe that this review makes a unique contribution to informing policy and practice by covering a comprehensive array of options for children-spanning both prevention and treatment, spanning both psychosocial and pharmacological interventions, and spanning developmental stages from prenatal through adolescence. By presenting this 'big picture', we hope to encourage policymakers and practitioners to implement effective interventions and to encourage them to consider the full array of options, thereby interrupting the planning and service fragmentation and gaps that can arise when only prevention or only treatment or only specific intervention types or only selected ages are considered. Our review also specifically updates a previous comprehensive review of ODD/CD interventions ${ }^{27}$ by covering a wider range of search years, encompassing studies that are both older and newer. We also believe that we have complemented previous reviews that had more narrow foci such as examining only prevention; ${ }^{28}$ only treatment ${ }^{29}$ or only specific intervention types. $^{30-33}$
Yet our review has limitations. We only reported on outcomes directly pertaining to $\mathrm{ODD} / \mathrm{CD}$. However, several prevention programmes showed other important benefits including: reducing child maltreatment (NurseFamily Partnership); ${ }^{35}$ reducing child suicidal ideation (Good Behavior Game) $)^{36}$ and improving adult employment (Perry Preschool). ${ }^{37}$ Likewise, some psychosocial treatments showed other benefits such as reducing substance use (Multisystemic Therapy) ${ }^{38}$ and reducing attention-deficit symptoms (Strongest Families). ${ }^{39}$ Another limitation pertains to the lack of attention in the prevention and psychosocial treatment studies to the issue of possible adverse effects. For example, interventions such as Nurse-Family Partnership entail greater family scrutiny, which may lead to adverse effects (from the family's perspective) such as additional child protection reporting; likewise, targeted programmes entail identifying children at risk, which may lead to potential stigma. Future prevention and psychosocial treatment research should attend to these issues. Our inclusion criteria for blinding also differed between psychosocial and medication studies, which may introduce bias favouring psychosocial studies. We took this approach to allow us to include a reasonable number of psychosocial intervention studies, where double blinding (and placebo controls) may not be feasible. As well, we required 
Table 4 Pharmacological treatment descriptions and evaluation findings

\begin{tabular}{|c|c|c|c|c|c|}
\hline $\begin{array}{l}\text { Medication } \\
\text { (daily dose)* }\end{array}$ & $\begin{array}{l}\text { Sample size } \\
\text { (country) }\end{array}$ & $\begin{array}{l}\text { Ages at post- } \\
\text { test }\end{array}$ & $\begin{array}{l}\text { Duration } \\
\text { (setting) }\end{array}$ & $\begin{array}{l}\text { Child behaviour } \\
\text { outcomes }\end{array}$ & $\begin{array}{l}\text { Child } \\
\text { adverse } \\
\text { events } t\end{array}$ \\
\hline $\begin{array}{l}\text { Carbamazepine } \\
(400-800 \mathrm{mg})^{\mathrm{w} 94}\end{array}$ & $\begin{array}{l}24 \\
\text { (USA) }\end{array}$ & $5-11$ years & $\begin{array}{l}6 \text { weeks } \\
\text { (hospital) }\end{array}$ & - 5 of 5 symptoms & $\begin{array}{l}\text { Weight gain } 77 \% \text {; dizziness } 54 \% \text {; headache } 46 \% \text {; } \\
\text { leucopenia } 46 \% \text {; rash } 46 \% \text {; diplopia } 38 \% \text {; drowsiness } 31 \% \text {; } \\
\text { nausea } 31 \% \text {; left study due to adverse events } 8 \%\end{array}$ \\
\hline $\begin{array}{l}\text { Haloperidol } \\
(1-12 \mathrm{mg})\end{array}$ & $\begin{array}{l}64 \\
\text { (USA) }\end{array}$ & $5-12$ years & $\begin{array}{l}4 \text { weeks } \\
\text { (hospital) }\end{array}$ & $\downarrow 5$ of 6 symptoms $\ddagger$ & $\begin{array}{l}\text { Any adverse event } 100 \% \text {; sedation } 80 \% \text {; dystonia } 50 \% \text {; } \\
\text { drooling } 30 \% \text {; tremor } 25 \% \text {; left study due to adverse events } \\
\text { not reported }\end{array}$ \\
\hline $\begin{array}{l}\text { Lithium } \\
(500-2000 \mathrm{mg})^{\text {w95 }}\end{array}$ & & & & $\downarrow 5$ of 6 symptoms $\ddagger$ & $\begin{array}{l}\text { Any adverse event } 81 \% \text {; weight gain not reported§; } \\
\text { abdominal pain } 33 \% \text {; headache } 29 \% \text {; left study due to } \\
\text { adverse events not reported }\end{array}$ \\
\hline $\begin{array}{l}\text { Lithium } \\
(600-2100 \mathrm{mg})^{\text {w96 }}\end{array}$ & $\begin{array}{l}55 \\
\text { (USA) }\end{array}$ & $5-12$ years & $\begin{array}{l}6 \text { weeks } \\
\text { (hospital) }\end{array}$ & $\downarrow 3$ of 6 symptoms & $\begin{array}{l}\text { Weight gain } 76 \% \text {; vomiting } 48 \% \text {; abdominal pain } 32 \% \text {; } \\
\text { tremor } 28 \% \text {; left study due to adverse events not reported }\end{array}$ \\
\hline $\begin{array}{l}\text { Lithium } \\
(900-2100 \mathrm{mg})^{\text {w97 }}\end{array}$ & $\begin{array}{l}40 \\
\text { (USA) }\end{array}$ & 9-17 years & $\begin{array}{l}4 \text { weeks } \\
\text { (hospital) }\end{array}$ & $\downarrow 3$ of 3 symptoms & $\begin{array}{l}\text { Weight gain } 85 \% \text {; nausea } 60 \% \text {; excessive thirst } 60 \% \text {; } \\
\text { urinary frequency } 55 \% \Uparrow \text {; vomiting } 55 \% \text {; abdominal pain } \\
35 \% \text {; headache } 30 \% \text {; tremor } 25 \% \text {; left study due to adverse } \\
\text { events } 0 \%\end{array}$ \\
\hline $\begin{array}{l}\text { Quetiapine } \\
(200-600 \mathrm{mg})^{\text {w98 }}\end{array}$ & $\begin{array}{l}19 \\
\text { (USA) }\end{array}$ & 12-17 years & $\begin{array}{l}6 \text { weeks } \\
\text { (clinic) }\end{array}$ & $\downarrow 2$ of 4 symptoms & $\begin{array}{l}\text { Irritability } 78 \% \text {; restlessness } 78 \% \text {; agitation } 67 \% \text {; anxiety } \\
67 \% \text {; sedation } 67 \% \text {; pacing } 44 \% \text {; social withdrawal } 44 \% \text {; } \\
\text { reduced energy } 33 \% \text {; reduced alertness } 33 \% \text {; weight gain } \\
33 \% \text {; tachycardia not reported } 1 \text {; left study due to adverse } \\
\text { events } 11 \%\end{array}$ \\
\hline $\begin{array}{l}\text { Risperidone }{ }^{* *} \\
(1.5-4 \mathrm{mg})^{\text {w99 }}\end{array}$ & $\begin{array}{l}38 \\
\text { (Netherlands) }\end{array}$ & 12-18 years ${ }^{\dagger}$ & $\begin{array}{l}6 \text { weeks } \\
\text { (hospital) }\end{array}$ & $\downarrow 1$ of 3 symptoms & 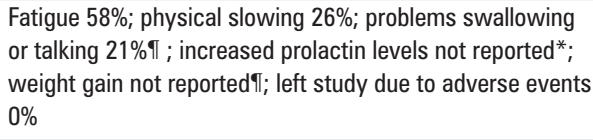 \\
\hline $\begin{array}{l}\text { Risperidone } \\
(0.75-1.5 \mathrm{mg})^{\mathrm{w} 100}\end{array}$ & $\begin{array}{l}20 \\
\text { (USA) }\end{array}$ & $6-14$ years & $\begin{array}{l}10 \text { weeks } \\
\text { (clinic) }\end{array}$ & $\downarrow 5$ of 6 symptoms & $\begin{array}{l}\text { Any side effect } 80 \% \text {; weight gain not reported } \% \text {; increased } \\
\text { appetite } 30 \% \text {; sedation } 30 \% \text {; left study due to adverse } \\
\text { events } 10 \%\end{array}$ \\
\hline 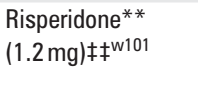 & $\begin{array}{l}119 \\
\text { (USA) }\end{array}$ & 5-12 years $t \dagger$ & $\begin{array}{l}6 \text { weeks } \\
\text { (clinic) }\end{array}$ & $\downarrow 3$ of 3 symptoms & 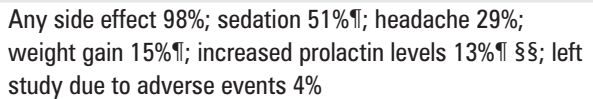 \\
\hline
\end{tabular}

- denotes no significant differences between intervention and placebo control groups; $\downarrow$ statistically significant reductions in symptoms.

* Reported doses include widest range that children received; dosing is not equivalent across medications.

†Adverse events only reported where $\geq 25 \%$ of children were affected or where significantly more children on medication versus placebo were affected; not all studies assessed whether medication and placebo adverse events differed significantly.

¥ln addition to both lithium and haloperidol showing significant symptom reductions over placebo on 5 of 6 measures, lithium showed significant benefits over haloperidol on 1 symptom measure.

§While authors did not report proportion of children experiencing weight gain, they noted that it was considerable (ranging from means of 31.7-35.0 kg).

IAdverse events experienced by significantly more children on medication than placebo.

${ }^{*}$ All children had below-average IOs.

††Ages reflect children eligible for the study.

$\ddagger \ddagger$ Average daily dose; range not reported.

$\S \S S$ ignificantly more boys (but not girls) experienced increased prolactin levels on medication versus placebo.

that psychosocial interventions have follow-up after post-test of at least 3 months, while only requiring post-test follow-up for medications. Still, risk-of-bias assessment for all included studies nevertheless confirmed more favourable profiles for medication compared with psychosocial studies, as acknowledged in the findings. Yet another limitation may arise from setting high thresholds for study inclusion. As a result, we do not discuss myriad interventions where we did not find RCT evaluations even though these interventions are likely being used-including some that may be ineffective. That said, we hope that our approach may serve as a pragmatic model for policymakers and practitioners. Namely, when interventions are lacking RCT evidence of effectiveness, they should only be used if there is a commitment to evaluating outcomes to ascertain the benefits for children.

Based on this review, the case for prevention appears to be particularly strong - with 13 programmes showing positive findings, many at long-term follow-up. Most successful programmes focused on children at risk, suggesting that targeted approaches may be particularly beneficial in ameliorating early adversities, arguably even constituting a form of 'proportionate universalism'. ${ }^{40}$ Many, furthermore, focused on the early years, suggesting greater benefits with 'upstream' approaches. Parent training was also a central feature of many successful programmes, suggesting that supporting parents is crucial.

Beyond positive child outcomes, US cost analyses have also been favourable for several effective prevention programmes, suggesting that they may pay for themselves. These US evaluations have factored in intervention costs as well as estimates of reduced additional service use across multiple sectors over 10-15 years or more-including criminal justice, social services, education and healthcare. Net benefits per participant have been suggested for: Good Behavior Game (US\$10 800, 2017 equivalency); Nurse-Family Partnership (US\$5 100, 2017 equivalency) and Parent Management Training-Oregon (US\$5 700, 2017 equivalency). ${ }^{12}$ Fast Track was also estimated to be cost-effective but only for the highest-risk children. ${ }^{41}$ For Perry Preschool, meanwhile, US estimates have suggested returns of 6-13 dollars for every programme 
dollar invested. ${ }^{42}$ Estimates will vary according to countries and methods used; such estimates may nevertheless be helpful in encouraging more widespread adoption of effective prevention programmes.

Yet even if effective prevention programmes are made widely available, they could not avert all new ODD or CD cases. Treatment will always be needed. According to this review, Incredible Years was notable for reducing ODD diagnoses, while Brief Intervention, Multidimensional Treatment Foster Care and Multisystemic Therapy reduced criminal activity. Psychosocial treatment benefits lasted from 1 to 8 years - considerable time in the life of a child. US cost analyses were also favourable for one effective programme: Multidimensional Treatment Foster Care. Factoring in programme costs as well as estimated lifetime reductions in the use of additional criminal justice, social, education and healthcare services, net benefits per participant have been suggested (US\$9 400, 2017 equivalency). ${ }^{12}$ (Costs analyses were also available for Incredible Years but none exclusively focused on the RCTs we reviewed.) Yet with only 5 psychosocial treatments showing effectiveness, new research is needed to delineate more options. We also concur with previous reviewers who have noted the modest effect sizes for most psychosocial treatments for ODD/ $\mathrm{CD}$, especially when treatments begin when children are older ${ }^{33}$ — such that more research is warranted, particularly with younger age groups.

In comparison, in the medication studies we reviewed, child benefits were short-term and frequently associated with important adverse events. Some of these adverse events were fairly rare and could likely be managed clinically. Yet these findings are still concerning given well-documented overprescribing to children ${ }^{23-26}$ _in a context where effective prevention programmes and psychosocial treatments are not widely available. ${ }^{1643}$ As well, prescriptions for behaviour problems have often involved 'off label' use - raising additional risks for children. ${ }^{44}$ The lack of long-term follow-up studies on these medications is a further cause for concern given the evidence amassing on cardiovascular, cognitive and other harms associated with the long-term use of antipsychotics such as risperidone and quetiapine in children. ${ }^{26} 45$ More research is therefore needed on pharmacological treatments for childhood ODD/CD, better delineating long-term risks and benefits.

On balance, most of the research evidence that we identified favours prevention. Yet substantial hurdles need to be overcome to implement this evidence. Foremost, in many wealthy countries, most health spending goes towards providing healthcare after problems are established. In the UK and Canada, for example, only approximately $5 \%$ of overall health spending is allocated to public health including prevention. ${ }^{17} 19$ Policymakers will need to take concerted leadership to shift these spending patterns, even by modest amounts. Yet some countries are excelling at improving children's mental health services, showing that the shortfalls and imbalances can be addressed. Australia, for example, has doubled the proportion of children with mental disorders who are receiving servicesfrom one-third in 1998 to two-thirds in 2014-by making significant new prevention and treatment investments. ${ }^{46}$ Beyond this, many prevention programmes require cross-sectoral collaboration for effective implementation. For instance, Classroom-Centered Intervention, Good Behavior Game and Fast Track were delivered in schools, involving teachers, while Nurse-Family Partnership was delivered in homes, involving public-health nurses. So novel collaborations among children's mental health agencies, public health agencies, schools and others will need to be established and sustained where these do not exist.

Making prevention a priority while ensuring the availability of effective treatments will also require concerted efforts from practitioner groups. Physicians - and their professional regulatory bodies_could take leadership in addressing medication overprescribing. Physicians as well as other practitioner groups including psychologists, social workers, nurses, teachers, child protection workers and school counsellors could engage in promoting effective prevention and psychosocial interventions for ODD/ CD. All practitioner groups could also take leadership in embracing intervention models that move the children's mental health field away from focussing only on individual approaches, reaching just one child at a time, towards also reaching many more children through population-level interventions, such as the effective prevention programmes highlighted here. Policymakers can assist with these efforts by funding effective interventions and by supporting practitioners to provide these.

Considerable RCT evidence favours prevention, according to this review. Effective prevention programmes should therefore be made widely available. Effective psychosocial treatments should also be provided for all children with ODD/CD. But medications should be a last resort given associated adverse events and only short-term evidence of benefits. While more research is needed, particularly on psychosocial interventions and on medications, policymakers and practitioners can nevertheless help children by acting on these findings now. Yet the aim is not to favour only prevention programmes, but rather, to achieve a balance of public investments - in effective interventions across the prevention and treatment continuum, so that all children in need are reached. The well-being of children and of populations is in the balance.

- References 48-104 will appear online only, and can be found in the supplementary appendix.

Contributors CW developed the idea for the review and wrote the manuscript. CS extracted relevant data, interpreted the data and contributed to the manuscript. CA and JLB conducted the literature searches, extracted relevant data and contributed to the manuscript. DY extracted relevant data and contributed to the manuscript. All authors approved the final version.

Funding This work was supported by the Canada Research Chairs Program (grant number 950-228413, dated 1 March 2013) and by the British Columbia Ministry of Children and Family Development (grant number SL00444S01, dated 11 April 2011, modified 30 December 2015)

Competing interests CW is coleading a randomised controlled trial on Nurse-Family Partnership, one of the interventions is discussed in this systematic review.

Provenance and peer review Not commissioned; externally peer reviewed.

\section{(6) OPEN ACCESS}

Open Access This is an Open Access article distributed in accordance with the Creative Commons Attribution Non Commercial (CC BY-NC 4.0) license, which permits others to distribute, remix, adapt, build upon this work non-commercially, and license their derivative works on different terms, provided the original work is properly cited and the use is non-commercial. See: http://creativecommons.org/licenses/ by-nc/4.0/

(C) Article author(s) (or their employer(s) unless otherwise stated in the text of the article) 2018. All rights reserved. No commercial use is permitted unless otherwise expressly granted.

- Additional material is published online only. To view please visit the journal online (http://dx.doi.org/10.1136/eb-2017-102862)

doi:10.1136/eb-2017-102862

Received 9 December 2017; Revised 19 March 2018; Accepted 26 March 2018

\section{REFERENCES}

1 American Psychiatric Association (APA). Diagnostic and Statistical Manual of Mental Disorders. $5^{\text {th }}$ Edition. Washington, DC: APA, 2013.

2 World Health Organization (WHO). International Statistical Classification of Diseases and Related Health Problems. 10th Revision: ICD-10. Geneva: WHO, 2016.

3 Polanczyk GV, Salum GA, Sugaya LS, et al. Annual research review: a meta-analysis of the worldwide prevalence of mental disorders in children and adolescents. J Child Psychol Psychiatry 2015;56:345-65.

4 McMahon RJ, Frick PJ. Evidence-based assessment of conduct problems in children and adolescents. J Clin Child Adolesc Psychol 2005;34:477-505.

5 Kessler RC, Berglund P, Demler 0, et al. Lifetime prevalence and age-of-onset distributions of DSM-IV disorders in the National Comorbidity Survey Replication. Arch Gen Psychiatry 2005;62:593-602. 
6 Rutter M, Moffitt TE, Caspi A. Gene-environment interplay and psychopathology: multiple varieties but real effects. J Child Psychol Psychiatry 2006;47:226-61.

7 Cohen MA, Piquero AR. New evidence on the monetary value of saving a high risk youth. J Quant Criminol 2009;25:25-49.

8 Costello EJ, Compton SN, Keeler G, et al. Relationships between poverty and psychopathology: a natural experiment. JAMA 2003;290:2023-9.

9 Reiss F. Socioeconomic inequalities and mental health problems in children and adolescents: a systematic review. Soc Sci Med 2013;90:24-31.

10 Caspi A, McClay J, Moffitt TE, et al. Role of genotype in the cycle of violence in maltreated children. Science 2002;297:851-4

11 Waddell C, Shepherd C, McLauchlin G. Creating mentally healthy communities, starting with children. In: The Canadian Population Health Initiative, eds. Mentally healthy communities: a collection of papers. Ottawa, ON: Canadian Institute for Health Information (CIHI), 2008:45-58.

12 Washington State Institute for Public Policy (WSIPP). Benefit-cost results. Updated 2017 www.wsipp.wa.gov/BenefitCost (accessed Nov 2017)

13 Gilbert R, Widom CS, Browne K, et al. Burden and consequences of child maltreatment in high-income countries. Lancet 2009;373:68-81.

14 Reading R, Bissell S, Goldhagen J, et al. Promotion of children's rights and prevention of child maltreatment. Lancet 2009;373:332-43.

15 Moreno C. Prevention in child and adolescent psychiatry: are we there yet? Eur Child Adolesc Psychiatry 2017;26:267-9.

16 Waddell C, Shepherd C, Schwartz C, et al. Child and youth mental disorders: prevalence and evidence-based interventions. Vancouver, BC: Children's Health Policy Centre, Faculty of Health Sciences, Simon Fraser University, 2014

17 Office for National Statistics. UK Health Accounts: 2015. Apr 2017. www.ons.gov. uk/releases/ukhealthaccounts2015 (accessed Nov 2017).

18 Martin AB, Hartman M, Benson J, et al. National Health Expenditure Accounts Team National health spending in 2014: faster growth driven by coverage expansion and prescription drug spending. Health Aff 2016:35:150-60.

19 CIHI. National health expenditure trends, 1975 to 2017. Ottawa, ON: CIHI, 2017.

20 Kieling C, Baker-Henningham $\mathrm{H}$, Belfer M, et al. Child and adolescent mental health worldwide: evidence for action. Lancet 2011;378:1515-25.

21 Whiteford HA, Degenhardt L, Rehm J, et al. Global burden of disease attributable to mental and substance use disorders: findings from the Global Burden of Disease Study 2010. Lancet 2013;382:1575-86.

22 WHO. Mental health atlas 2014. Geneva: WHO, 2015.

23 Rani F, Murray ML, Byrne PJ, et al. Epidemiologic features of antipsychotic prescribing to children and adolescents in primary care in the United Kingdom. Pediatrics 2008;121:1002-9.

24 Alessi-Severini S, Biscontri RG, Collins DM, et al. Ten years of antipsychotic prescribing to children: a Canadian population-based study. Can J Psychiatry 2012:57:52-8.

25 Ronsley R, Scott D, Warburton WP, et al. A population-based study of antipsychotic prescription trends in children and adolescents in British Columbia, from 1996 to 2011 Can J Psychiatry 2013;58:361-9.

26 Hauck TS, Lau C, Wing LLF, et al. ADHD treatment in primary care: demographic factors, medication trends, and treatment predictors. Can J Psychiatry 2017;62:393-402.

27 Connor DF, Carlson GA, Chang KD, et al. Juvenile maladaptive aggression: a review of prevention, treatment, and service configuration and a proposed research agenda. J Clin Psychiatry 2006;67:808-20.

28 Waddell C, Hua JM, Garland OM, et al. Preventing mental disorders in children: a systematic review to inform policy-making. Can J Public Health 2007;98:166-73.
29 National Collaborating Centre for Mental Health. Antisocial behaviour and conduct disorders in children and young people: the NICE guideline on recognition, intervention and management. Great Britain: The British Psychological Society and The Royal College of Psychiatrists, 2013.

30 Woolfenden SR, Williams K, Peat JK. Family and parenting interventions for conduct disorder and delinquency: a meta-analysis of randomised controlled trials. Arch Dis Child 2002;86:251-6

31 Barlow J, Smailagic N, Bennett C, et al. Individual and group based parenting programmes for improving psychosocial outcomes for teenage parents and their children. Cochrane Database Syst Rev 2011:74:CD002964.

32 Sanders MR, Kirby JN, Tellegen CL, et al. The Triple P-Positive Parenting Program: a systematic review and meta-analysis of a multi-level system of parenting support. Clin Psychol Rev 2014;34:337-57.

33 Bakker MJ, Greven CU, Buitelaar JK, et al. Practitioner Review: psychological treatments for children and adolescents with conduct disorder problems - a systematic review and meta-analysis. J Child Psychol Psychiatry 2017:58:4-18.

34 Higgins JPT, Green S. Cochrane handbook for systematic reviews of interventions, version 5.1.0. Updated Mar 2011 www.handbook-5-1.cochrane.org laccessed Nov 2017)

35 Olds DL, Eckenrode J, Henderson CR, et al. Long-term effects of home visitation on maternal life course and child abuse and neglect. Fifteen-year follow-up of a randomized trial. JAMA 1997;278:637-43.

36 Wilcox HC, Kellam SG, Brown $\mathrm{CH}$, et al. The impact of two universal randomized first- and second-grade classroom interventions on young adult suicide ideation and attempts. Drug Alcohol Depend 2008;95 Suppl 1:S60-S73.

37 Schweinhart LJ. Long-term follow-up of a preschool experiment. J Exp Criminol 2013;9:389-409.

38 Henggeler SW, Clingempeel WG, Brondino MJ, et al. Four-year follow-up of multisystemic therapy with substance-abusing and substance-dependent juvenile offenders. J Am Acad Child Adolesc Psychiatry 2002;41:868-74.

39 McGrath PJ, Lingley-Pottie P, Thurston C, et al. Telephone-based mental health interventions for child disruptive behavior or anxiety disorders: randomized trials and overall analysis. J Am Acad Child Adolesc Psychiatry 2011;50:1162-72.

40 Marmot M, Allen J, Goldblatt P, et al. Fair society, healthy lives: a strategic review of health inequalities in England post-2010. London, UK: Department of Epidemiology and Public Health, University College London, 2010.

41 Foster EM, Jones D; Conduct Problems Prevention Research Group. Can a costly intervention be cost-effective?: An analysis of violence prevention. Arch Gen Psychiatry 2006:63:1284-91.

42 Nores M, Belfield CR, Barnett WS, et al. Updating the economic impacts of the High/ Scope Perry Preschool Program. Educ Eval Policy Anal 2005:27:245-61.

43 Waddell C, McEwan K, Peters RD, et al. Preventing mental disorders in children: a public health priority. Can J Public Health 2007;98:174-8.

44 Stafford RS. Off-label use of drugs and medical devices: a review of policy implications. Clin Pharmacol Ther 2012;91:920-5.

45 Panagiotopoulos C, Ronsley R, Davidson J. Increased prevalence of obesity and glucose intolerance in youth treated with second-generation antipsychotic medications. Can J Psychiatry 2009:54:743-9.

46 Lawrence D, Johnson S, Hafekost J, et al. The mental health of children and adolscents: report on the second Australian Child and Adolescent Survey of Mental Health and Wellbeing. Canberra 2015.

47 Moher D, Liberati A, Tetzlaff J, et al. PRISMA Group. Preferred reporting items for systematic reviews and meta-analyses: the PRISMA statement. PLoS Med 2009; 6:e1000097. 